\title{
Fluid-Structural Coupling Effects on the Dynamics of Mistuned Bladed Disks
}

\author{
Zhijiang He* and Bogdan I. Epureanu \\ University of Michigan, Ann Arbor, Michigan 48109 \\ and \\ Christophe Pierre \\ McGill University, Montreal, Quebec H3A-2K6, Canada \\ DOI: $\underline{10.2514 / 1.23809}$
}

\begin{abstract}
Mistuning changes the vibration of bladed disks dramatically. Various aeroelastic models have been used to investigate the free vibration and forced response problems of mistuned bladed disks. Most of these models used simplified structural and/or aerodynamic models. The traditional way to incorporate the aerodynamic coupling in the high-fidelity structural models is to use the cantilever-blade normal modes to calculate the unsteady aerodynamic forces. In this paper, a new reduced-order modeling approach is developed by using the tuned system modes to calculate the unsteady aerodynamic forces directly. This new approach is applied to an industrial rotor. The results show that aerodynamic coupling has significant effects on the vibration of bladed disks for the case studied. Also, constraint modes are needed to yield accurate results if cantilever-blade normal modes are used to calculate the unsteady aerodynamic forces. However, using the tuned system modes to calculate the unsteady aerodynamic forces saves a significant amount of computation time compared to the method using both cantilever-blade normal modes and constraint modes.
\end{abstract}

\section{Nomenclature}

\section{$\boldsymbol{A}$}

C

E

$f, \boldsymbol{F}$

$h$

j

$\boldsymbol{K}, \boldsymbol{M}$

$N_{B}$

P, $Q$

$p$

$Q$

$q$

S

$T$

U

$\delta \boldsymbol{K}, \delta \boldsymbol{M}=$ physical mistuned stiffness and mass matrices

$\gamma=$ structural damping

$\gamma_{c} \quad=$ ratio of specific heats

$\rho \quad=$ flow density

$\sigma \quad=$ interblade phase angle

$\boldsymbol{\Phi} \quad=$ system and blade mode shapes

$\phi \quad=$ velocity potential of the flow

$\boldsymbol{\Psi}=$ blade constraint mode shape

$\omega=$ complex frequency
Received 11 March 2006; revision received 28 November 2006; accepted for publication 23 November 2006. Copyright (C) 2006 by Zhijiang He, Bogdan I. Epureanu and Christophe Pierre. Published by the American Institute of Aeronautics and Astronautics, Inc., with permission. Copies of this paper may be made for personal or internal use, on condition that the copier pay the $\$ 10.00$ per-copy fee to the Copyright Clearance Center, Inc., 222 Rosewood Drive, Danvers, MA 01923; include the code 0001-1452/07 $\$ 10.00$ in correspondence with the CCC.

*Graduate Research Assistant, Department of Mechanical Engineering, 2350 Hayward Street; hez@umich.edu. Student Member AIAA.

Assistant Professor, Department of Mechanical Engineering, 2350 Hayward Street; epureanu@umich.edu. Member AIAA.

Dean, Faculty of Engineering, 817 Sherbrooke Street; christophe. pierre@mcgill.ca. Senior Member AIAA.

\author{
Subscripts \\ $T=$ total flow density and pressure \\ $\Gamma \quad=$ blade part of mode shape \\ Superscripts \\ $a=$ aerodynamic quantity \\ $\mathrm{CB}, S=$ cantilevered-blade modes and system modes \\ $M, T=$ mistuned and tuned \\ $n=$ steps of iterative calculations \\ syn $\quad=$ in the synthetic modal space \\ $0=$ structural-only quantities \\ $* \quad=$ Hermitian of a complex matrix
}

\section{Introduction}

$\mathbf{R}$ EDUCING vibrations, and in particular the resonant forced response of bladed disks, is an important concern for the turbomachinery industry. Cyclic symmetry is a convenient assumption for the analysis of (tuned) bladed disks. However, cyclic symmetry does not hold for mistuned disks, which exhibit small differences among sectors. Mistuning is unavoidable in practice due to manufacturing tolerances and in-service wear and tear, and can cause a drastic increase of the forced response. Hence, the effects of mistuning on the vibration of turbomachinery rotors have been extensively investigated (e.g., Tobias and Arnold [1]).

In early work, simplified models with only several degrees of freedom (DOF) were used to study the dynamics of mistuned bladed disks [2-4]. Although such simplified models, like lumped parameter models, are convenient and insightful to investigate the effects of major system parameters (such as mistuning, structural and aerodynamic coupling), they are not accurate enough to study real/ practical bladed disks. Models based on the finite element method (FEM) can represent the actual bladed disks accurately. However, the large number of DOF (usually several millions) of the FEM models makes the computation time practically not affordable. Hence, different reduced-order models (ROM) have been developed [5-12]. Current high-fidelity structural models reduce the computation time greatly compared to the original FEM model. Moreover, various fluid dynamic models have been developed to 
calculate with high speed the unsteady pressure on the blades [1317] by (usually) assuming linearity of the unsteady pressure with respect to the displacements of the blades.

Severe mode localization and excessive vibration amplitude of bladed disks are major detrimental effects of mistuning. Previous work has shown that interblade structural coupling is a critical factor affecting the dynamics of mistuned bladed disks. In operating conditions, bladed disks are always interacting with flows, and hence, bladed disks exhibit both structural and aerodynamical coupling. Thus, aeroelastic calculations are necessary for accurate predictions. Although compact and accurate structural reduced-order models have been developed, only a few studies of the dynamics of mistuned bladed disks have been conducted with considerations of aerodynamic forces. Traditionally, the aerodynamic stiffness matrix was calculated using the blade normal modes with/without constraint modes. For example, Kaza and Kielb [18,19] used a beam structural model and a two-dimensional aerodynamic model to study the vibrations and flutter of mistuned bladed disks. Pierre and Murthy [20] and Pierre et al. [21] developed a perturbation method to investigate the aeroelastic mode localization and eigenstructure transformations. Sadeghi and Liu [22] studied the phase mistuning and frequency mistuning effects on two-dimensional cascade flutter. Gerolymos [23] calculated the tuned aeroelastic modes using an iterative process over frequency-dependent unsteady aerodynamic forces for a shroudless bladed disk. Moyroud et al. [24] developed a similar iteration process using different structural and aerodynamic computer codes, and calculated the aeroelastic tuned eigenvalues for bladed disks with and without shrouds. In these latter two methods $[23,24]$, the aerodynamic code used the tuned system modes to calculate the unsteady aerodynamic forces. During the iterative process, Gerolymos [23] used a mode modification technique to update the new eigenvalues and eigenvectors, and Moyroud et al. [24] solved the eigenvalue problem directly. Seinturier et al. [25] adopted the structural model developed by Bladh et al. [6, 7] to calculate the forced response of mistuned bladed disks. Because that structural model is substructured into blade and disk components, cantilever-blade normal modes and constraint modes were used to calculate the unsteady aerodynamic forces [25]. The constraint modes are obtained by enforcing unit displacements on interface DOF successively. Kielb et al. [26,27] used the fundamental mistuning model (FMM) developed by Feiner and Griffin [11] to investigate the flutter and forced response problems of mistuned bladed disks. Although FMM uses the tuned system modes as the modal basis, cantilever-blade normal modes are still used to calculate the unsteady aerodynamic forces [26,27].

Distinct from these previous studies, the current paper discusses a new method to incorporate the aerodynamic coupling into a highfidelity structural reduced-order model by using the tuned system modes directly to calculate the unsteady aerodynamic stiffness matrix, and by employing iterations over the eigenvalues of the system to obtain accurate results. A new fluid-structure coupling method in the modal space is developed to incorporate the aerodynamic effects into a compact and accurate ROM. The component mode mistuning (CMM) method [8] is used for the structural model, and a quasi-3-D unsteady aerodynamic code is employed to calculate the unsteady aerodynamic forces. Traditionally, the cantilever-blade normal modes (and the constraint modes) have been used to calculate the aerodynamic stiffness matrix $[20,21,25-27]$. However, in the CMM model, the tuned system modes are used as the modal basis. Herein, the tuned system modes are used to calculated the aerodynamic stiffness matrix iteratively over the eigenvalues of the system. The results using the traditional method and the new method are compared. It is found that, for the case studied, there are notable differences between the results using the new model and the traditional method with cantilever-blade normal modes only. To achieve accurate results, constraint modes are necessary. However, the inclusion of constraint modes in the aerodynamic calculation increases significantly the computation time compared to using the tuned system modes directly.

This paper is organized as follows: In the next two sections, the CMM structural model and the quasi-3-D aerodynamic model are introduced. The methods to incorporate the aerodynamic coupling into the CMM model using the cantilever-blade normal modes and the tuned system modes are presented in the following section. The two methods are applied to an industrial rotor. Results and discussions are presented in the following section. Finally, the last section summarizes the conclusions of this work.

\section{Structural Reduced-Order Model}

The common method for bladed disks is to conduct FEM analysis for only one sector based on cyclic symmetry. However, when the cyclic symmetry is destroyed by mistuning, the analysis of the whole model is needed if FEM method is employed. Herein, an efficient and precise reduced-order modeling method developed by Lim et al. [] for mistuned bladed disks is used. A group of tuned system modes are used as the modal space of the whole (mistuned) system. The major advantage of this reduced-order model is the projection of the deviation of cantilever-blade stiffness onto the tuned system modal space. For brevity, only the synthesized equations in the reduced modal space are presented here. The details of the reduced-order model can be found in the original paper (by Lim et al. [8]). The hybrid-interface component mode synthesis (CMS) method is employed in this model. As shown in Fig. 1, the mistuned bladed disk is partitioned into two components: a free-interface component (the tuned bladed disk) and a fixed-interface component (the blade mistuning). The fixed-interface component is a virtual component instead of a physical component because it is the difference between the mistuned system and the nominal tuned system. Under the assumption that the mistuned modes for a system with small mistuning can be captured by the tuned system modes alone when the tuned system modes have close frequencies (within a small range [ $\underline{8}-$ $10]$ ), the ROM can be constructed by using only the tuned system modes (which are obtained by conducting cyclic symmetry analysis of one sector). The synthesized equations for the eigenvalue problem and (separately) the forced response problem can be expressed as [8]

$$
\begin{gathered}
{\left[(1+j \gamma) \boldsymbol{K}^{\mathrm{syn}}+\boldsymbol{K}^{a}-\omega^{2} \boldsymbol{M}^{\mathrm{syn}}\right] \boldsymbol{q}_{\phi}^{S}=\mathbf{0}} \\
{\left[(1+j \gamma) \boldsymbol{K}^{\mathrm{syn}}+\boldsymbol{K}^{a}-\omega^{2} \boldsymbol{M}^{\mathrm{syn}}\right] \boldsymbol{q}_{\phi}^{S}=\boldsymbol{\Phi}_{\Gamma}^{S, 0 *} \boldsymbol{f}}
\end{gathered}
$$

where matrices $\boldsymbol{M}^{\text {syn }}$ and $\boldsymbol{K}^{\text {syn }}$ are given by

$$
\boldsymbol{M}^{\mathrm{syn}}=\boldsymbol{I}+\boldsymbol{\Phi}_{\Gamma}^{S, 0 *} \delta \boldsymbol{M} \boldsymbol{\Phi}_{\Gamma}^{S, 0}, \quad \boldsymbol{K}^{\mathrm{syn}}=\boldsymbol{\Lambda}+\boldsymbol{\Phi}_{\Gamma}^{S, 0 *} \delta \boldsymbol{K} \boldsymbol{\Phi}_{\Gamma}^{S, 0}
$$

\section{Frequency Domain Unsteady Aerodynamic Model}

A quasi-three-dimensional model of a cascade operating in an inviscid, irrotational, and isentropic flow is employed based on the full-potential equation using a Galerkin formulation [13] and by considering the variation of stream tube heights [28]. Consider the flow between two neighboring stream surfaces. The velocity vector may be expressed as the gradient of the scalar velocity potential $\hat{\phi}$. The conservation of mass can be expressed as

$$
\frac{\partial(\hat{\rho} h)}{\partial t}+\nabla \cdot(\hat{\rho} \nabla \hat{\phi} h)=0
$$

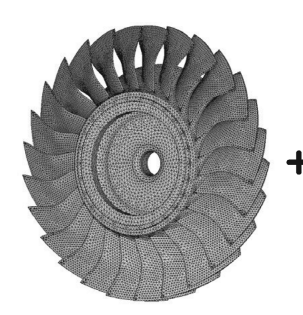

Complete tuned bladed disk $\left(\mathbf{M}^{\mathrm{S}}, \mathbf{K}^{\mathrm{S}}\right)$

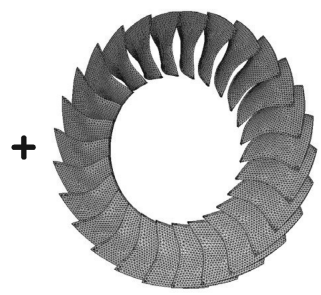

Blade mistuning components $\left(\mathbf{M}^{\delta}, \mathbf{K}^{\delta}\right)$
Fig. 1 Substructuring of a mistuned bladed disk. 
where ^denotes the full solution, including steady and unsteady components. For an isentropic flow, the density and the pressure can be expressed as

$$
\begin{aligned}
& \hat{\rho}=\rho_{T}\left\{1-\frac{\gamma_{c}-1}{C_{T}^{2}}\left[\frac{1}{2}(\nabla \hat{\phi})^{2}+\frac{\partial \hat{\phi}}{\partial t}\right]\right\}^{1 /\left(\gamma_{c}-1\right)}, \\
& \hat{p}=p_{T}\left\{1-\frac{\gamma_{c}-1}{C_{T}^{2}}\left[\frac{1}{2}(\nabla \hat{\phi})^{2}+\frac{\partial \hat{\phi}}{\partial t}\right]\right\}^{\gamma_{c} /\left(\gamma_{c}-1\right)}
\end{aligned}
$$

where $\hat{p}$ is the (complete steady and unsteady) pressure. Equation (3) can be transformed by applying a variational principle $[13,29]$. Namely, the velocity potential, which satisfies Eq. (3) in a simpleconnected domain $D$, renders extremum of the functional $\Pi$ given by

$$
\Pi=\frac{1}{T} \int_{T} \iint_{D} \hat{p} h \mathrm{~d} x \mathrm{~d} y \mathrm{~d} t+\frac{1}{T} \int_{T} \oint \hat{Q} \hat{\phi} h \mathrm{~d} s \mathrm{~d} t
$$

where $\hat{Q}$ is the prescribed mass flux on the boundary.

The steady flow in each stream tube is calculated first. Then, the unsteady flow is linearized about the steady flow under the assumption that the unsteady flow induced by the motion of the blades is a small perturbation to the steady flow. Only one passage between two neighboring blades is solved by assuming that the flow is periodic along the circumferential direction. Hence, the velocity potential can be expressed as the sum of a steady potential $\Phi$ and the real part of an unsteady periodic potential $\phi$, i.e., $\hat{\phi}(x, y, t)=$ $\Phi(x, y)+\Re\left[\phi(x, y) e^{j \omega t}\right]$, with $\phi \ll \Phi$ and $\Re$ denoting the real part.

\section{Fluid-Structure Coupling Models}

Fluid-Structure Coupling Using Blade Modes

In previous work (Lim et al. [8] ), the stiffness mistuning projection is implemented by using the following modal transformation:

$$
\boldsymbol{\Phi}_{\Gamma}^{S, 0}=\boldsymbol{I} \otimes\left[\boldsymbol{\Phi}^{\mathrm{CB}} \boldsymbol{\Psi}^{\mathrm{CB}}\right]\left[\begin{array}{l}
\boldsymbol{q}_{\phi}^{\mathrm{CB}} \\
\boldsymbol{q}_{\psi}^{\mathrm{CB}}
\end{array}\right]=\left(\boldsymbol{I} \otimes \boldsymbol{U}^{\mathrm{CB}}\right) \boldsymbol{q}^{\mathrm{CB}}
$$

where $\otimes$ denotes the Kronecker product [30], and

$$
\boldsymbol{U}^{\mathrm{CB}}=\left[\begin{array}{ll}
\boldsymbol{\Phi}^{\mathrm{CB}} & \boldsymbol{\Psi}^{\mathrm{CB}}
\end{array}\right], \quad\left(\boldsymbol{q}^{\mathrm{CB}}\right)^{*}=\left[\begin{array}{ll}
\left(\boldsymbol{q}_{\phi}^{\mathrm{CB}}\right)^{*} & \left(\boldsymbol{q}_{\psi}^{\mathrm{CB}}\right)^{*}
\end{array}\right]
$$

The constraint modes are obtained by enforcing unit displacements on each DOF at the interface successively, while keeping other DOFs at the interface fixed. The purpose of $\Psi^{\mathrm{CB}}$ is to describe the displacements at the boundaries between components of the bladed disk, such as blade-disk boundary and shroud-to-shroud boundary.

The projection used in Eq. (4) can be used to calculate the aerodynamic stiffness matrix $\overline{\boldsymbol{K}^{a}}$ also. First, the aerodynamic stiffness coupling coefficients in the complex cyclic constraint and cantilever-blade normal modal coordinates $\tilde{\boldsymbol{A}}$ are calculated. The modal transformation between $\tilde{\boldsymbol{A}}$ and the aerodynamic stiffness coupling coefficients in the physical coordinates $\boldsymbol{A}$ can be expressed as [ㅁ]

$$
\begin{aligned}
\tilde{\boldsymbol{A}} & =\left(\boldsymbol{I} \otimes \boldsymbol{U}^{\mathrm{CB} *}\right)\left(\boldsymbol{E}^{*} \otimes \boldsymbol{I}\right) \boldsymbol{A}(\boldsymbol{E} \otimes \boldsymbol{I})\left(\boldsymbol{I} \otimes \boldsymbol{U}^{\mathrm{CB}}\right) \\
& =\left(\boldsymbol{E}^{*} \otimes \boldsymbol{I}\right)\left(\boldsymbol{I} \otimes \boldsymbol{U}^{\mathrm{CB} *}\right) \boldsymbol{A}\left(\boldsymbol{I} \otimes \boldsymbol{U}^{\mathrm{CB}}\right)(\boldsymbol{E} \otimes \boldsymbol{I})
\end{aligned}
$$

Therefore, the aerodynamic stiffness coupling matrix in the tuned structure-only system modal coordinates can be expressed [using Eqs. (4) and (ㄷ)] in the following form:

$$
\begin{aligned}
\boldsymbol{K}^{a} & =\boldsymbol{\Phi}_{\Gamma}^{S, 0 *} \boldsymbol{A} \boldsymbol{\Phi}_{\Gamma}^{S, 0}=\boldsymbol{q}^{\mathrm{CB} *}\left(\boldsymbol{I} \otimes \boldsymbol{U}^{\mathrm{CB} *}\right) \boldsymbol{A}\left(\boldsymbol{I} \otimes \boldsymbol{U}^{\mathrm{CB}}\right) \boldsymbol{q}^{\mathrm{CB}} \\
& =\boldsymbol{q}^{\mathrm{CB} *}(\boldsymbol{E} \otimes \boldsymbol{I}) \tilde{\boldsymbol{A}}\left(\boldsymbol{E}^{*} \otimes \boldsymbol{I}\right) \boldsymbol{q}^{\mathrm{CB}}
\end{aligned}
$$

For every mode shape $\boldsymbol{U}_{m}^{\mathrm{CB}}$ (i.e., for every column $m$ of $\boldsymbol{U}^{\mathrm{CB}}$ ), the unsteady pressure distribution on the blade $p_{m, i}^{\mathrm{CB}}$ is calculated for every interblade phase angle $\sigma_{i}$ of index $i$, which is given by $\sigma_{i}=2 \pi i / N_{B}$ (for $\left.i=0,1, \ldots, N_{B}-1\right)$. Note that $\sigma_{i}$ and $\sigma_{N_{B}+i}$ correspond to the same interblade phase angle. Hence, $\tilde{\boldsymbol{A}}$ is a block diagonal matrix where each block relates to a specific interblade phase angle index, and

$$
\tilde{\boldsymbol{A}}=\left[\begin{array}{llll}
\tilde{\boldsymbol{A}}^{0} & & & \\
& \tilde{\boldsymbol{A}}^{1} & & \\
& & \ddots & \tilde{\boldsymbol{A}}^{N_{B}-1}
\end{array}\right]
$$

The elements $\tilde{\boldsymbol{A}}_{m n}^{i}$ of the diagonal block $\tilde{\boldsymbol{A}}^{i}$ can be obtained by integrating the dot product of the $m$ th mode $\boldsymbol{U}_{m}^{C B}$ with the $n$th corresponding force in the direction of the local normal $\boldsymbol{n}$ as follows:

$$
\tilde{\boldsymbol{A}}_{m n}^{i}=\int_{A} \boldsymbol{U}_{m}^{\mathrm{CB} *} \cdot p_{n, i}^{\mathrm{CB}} \boldsymbol{n} \mathrm{d} A, \quad m, n=1,2, \ldots, N_{U}
$$

where $N_{U}$ is the number of modes in $\boldsymbol{U}_{m}^{\mathrm{CB}}$. The number of constraint modes are usually much larger than the number of cantilever-blade normal modes retained in $\boldsymbol{U}_{m}^{\mathrm{CB}}$. Hence, for bladed disks without shrouds, the constraint modes are usually neglected [8]. In this scenario, the dimension of $\tilde{\boldsymbol{A}}_{m n}^{i}$ in Eq. (8) is the number of cantilevered-blade modes selected $N_{\mathrm{CB}}$, and the modal transformations shown in Eqs. (ㅁ-ㅁ) successively become [ㅁ]

$$
\begin{gathered}
\boldsymbol{\Phi}_{\Gamma}^{S, 0}=\left(\boldsymbol{I} \otimes \boldsymbol{\Phi}^{\mathrm{CB}}\right) \boldsymbol{q}_{\phi}^{\mathrm{CB}} \\
\tilde{\boldsymbol{A}}=\left(\boldsymbol{I} \otimes \boldsymbol{\Phi}^{\mathrm{CB} *}\right)\left(\boldsymbol{E}^{*} \otimes \boldsymbol{I}\right) \boldsymbol{A}(\boldsymbol{E} \otimes \boldsymbol{I})\left(\boldsymbol{I} \otimes \boldsymbol{\Phi}^{\mathrm{CB}}\right) \\
=\left(\boldsymbol{E}^{*} \otimes \boldsymbol{I}\right)\left(\boldsymbol{I} \otimes \boldsymbol{\Phi}^{\mathrm{CB} *}\right) \boldsymbol{A}\left(\boldsymbol{I} \otimes \boldsymbol{\Phi}^{\mathrm{CB}}\right)(\boldsymbol{E} \otimes \boldsymbol{I}) \\
\boldsymbol{K}^{a}=\boldsymbol{\Phi}_{\Gamma}^{S, 0 *} \boldsymbol{A} \boldsymbol{\Phi}_{\Gamma}^{S, 0}=\boldsymbol{q}_{\phi}^{\mathrm{CB} *}\left(\boldsymbol{I} \otimes \boldsymbol{\Phi}^{\mathrm{CB} *}\right) \boldsymbol{A}\left(\boldsymbol{I} \otimes \boldsymbol{\Phi}^{\mathrm{CB}}\right) \boldsymbol{q}_{\phi}^{\mathrm{CB}} \\
=\boldsymbol{q}_{\phi}^{\mathrm{CB} *}(\boldsymbol{E} \otimes \boldsymbol{I}) \tilde{\boldsymbol{A}}\left(\boldsymbol{E}^{*} \otimes \boldsymbol{I}\right) \boldsymbol{q}_{\phi}^{\mathrm{CB}}
\end{gathered}
$$

\section{Fluid-Structure Coupling Using System Modes}

A natural other choice for calculating the aerodynamic stiffness matrix is to employ the same projection as the one used for mistuning, i.e., by using the approach shown in the preceding subsection. However, for the cases where the constraint modes are needed to calculate the aerodynamic stiffness matrix (e.g., bladed disks with shrouds), the computation time is likely to become formidable because the unsteady pressure distribution has to be calculated for every constraint mode and every interblade phase angle index. Moreover, the aerodynamic calculation is sensitive to the blade motion, and consequently the small errors caused by the modal projection [similar to the one shown in Eq. (9)] may lead to large errors in the resulting aerodynamic stiffness matrix (expressed in the structure-only tuned system modal coordinates). To overcome these difficulties, tuned (structure-only) system modes are used directly to calculated the aerodynamic stiffness matrix. Because these modes are structure-only modes, iterations over frequencies are needed because the aerodynamic stiffness matrix $\boldsymbol{K}^{a}$ in Eq. (1) is dependent on the frequencies. From Eq. (1), the aeroelastic system modes can be expressed as

$$
\boldsymbol{\Phi}_{\Gamma, i}^{S, n}=\sum_{r=1}^{N_{S}} Q_{\phi, r i}^{S, n} \boldsymbol{\Phi}_{\Gamma, r}^{S, 0}, \quad i=1,2, \ldots, N_{S}
$$

or in the matrix form as

$$
\boldsymbol{\Phi}_{\Gamma}^{S, n}=\boldsymbol{\Phi}_{\Gamma}^{S, 0} \boldsymbol{Q}_{\phi}^{S, n}
$$

where the superscript $n$ denotes results after $n$ steps of iteration, $N_{S}$ is the number of tuned structure-only system modes used in the component mode mistuning model, and the matrix $\boldsymbol{Q}_{\phi}^{S, n}$ is given by

$$
\boldsymbol{Q}_{\phi}^{S, n}=\left[\begin{array}{llll}
\boldsymbol{q}_{\phi, 1}^{S, n} & \boldsymbol{q}_{\phi, 2}^{S, n} & \ldots & \boldsymbol{q}_{\phi, N_{S}}^{S, n}
\end{array}\right]
$$

For a tuned system, the aeroelastic modes have constant interblade phase angles, whereas no constant interblade phase angle exists in 
the mistuned aeroelastic modes. However, the aerodynamic calculation requires periodic boundary conditions. To address this issue and calculate the converged aerodynamic stiffness matrix, two different methods are proposed: one for tuned systems and the other for mistuned systems. In the first step of both methods, structure-only mode shapes and (complex) eigenvalues $\omega^{0}$ are computed. Then, at every iteration step $n$, the aerodynamic stiffness matrix (expressed in the tuned structure-only modal coordinates) $\boldsymbol{K}^{a, n}$ is obtained using the eigenvalues $\omega^{n-1}$ calculated in the previous iteration step, and new eigenvalues $\omega^{n}$ are computed using $\boldsymbol{K}^{a, n}$. Iterations are performed until convergence in the aerodynamic stiffness matrix is achieved. This is distinct from classical flutter calculations where, for example, iterations are needed to determine the flutter speed. Here, the aeroelastic eigenvalues and eigenmodes for a particular flow condition (which is before flutter) are of interest. The iterations needed are performed over the eigenvalues (of unconverged aeroelastic system) because the aerodynamic stiffness matrix is dependent on these eigenvalues.

\section{Tuned Case}

Because every tuned aeroelastic system mode has a specific interblade phase angle, the corresponding unsteady pressure distribution on a blade has the same interblade phase angle also. Hence, the aerodynamic stiffness matrix in the tuned aeroelastic system modal coordinates $\hat{\boldsymbol{A}}^{T, n}$ may be expressed in the form of a block diagonal matrix as

$$
\hat{\boldsymbol{A}}^{T, n}=\left[\begin{array}{cccc}
\hat{\boldsymbol{A}}_{0}^{T, n} & & & \\
& \hat{\boldsymbol{A}}_{1}^{T, n} & & \\
& & \ddots & \hat{\boldsymbol{A}}_{N_{B}-1}^{T, n}
\end{array}\right]
$$

Unlike Eq. (7), the dimensions of the blocks $\hat{\boldsymbol{A}}_{i}^{T, n}$ in Eq. (15) can be different for different $i$, and depend on the number of tuned structureonly system modes used in the CMM model that relate to the $i$ th interblade phase angle $\sigma_{i}$. The aerodynamic stiffness matrix in the tuned structure-only system modal coordinates $\boldsymbol{K}^{a, n}$ can be calculated by using the modal transformation shown in Eq. (13). One obtains

$$
\boldsymbol{K}^{a, n}=\boldsymbol{P}^{n *} \hat{\boldsymbol{A}}^{T, n} \boldsymbol{P}^{n}
$$

where $\boldsymbol{P}^{n}=\left(\boldsymbol{Q}_{\phi}^{S, n}\right)^{-1}$ is the modal transformation from the tuned aeroelastic system modes to the tuned structure-only system modes.

\section{Mistuned Case}

Because of the linearity of the unsteady aerodynamic model, the unsteady aerodynamic forces exerted on the blade due to the $i$ th mistuned aeroelastic system mode $\boldsymbol{\Phi}_{\Gamma, i}^{S, n}$ can be expressed as

$$
\boldsymbol{F}_{i}^{S, n}=\sum_{r=1}^{N_{S}} Q_{\phi, r i}^{S, n} \boldsymbol{F}_{i, r}^{S, 0}, \quad i=1,2, \ldots, N_{S}
$$

Note that the mode $\boldsymbol{\Phi}_{i, r}^{S, 0}$ is vibrating with the $i$ th mistuned system natural frequency related to the force $\boldsymbol{F}_{i}^{S, n}$. Hence, the forces $\boldsymbol{F}_{i, r}^{S, 0}$ are different for distinct $\boldsymbol{F}_{i}^{S, n}$. The elements of the aerodynamic stiffness matrix (in the mistuned aeroelastic system modal coordinates) $\hat{\boldsymbol{A}}^{M, n}$ can be obtained as

$$
\begin{gathered}
\hat{\boldsymbol{A}}_{i j}^{M, n}=\boldsymbol{\Phi}_{\Gamma, i}^{S, n *} \boldsymbol{F}_{j}^{S, n}=\left(\sum_{r=1}^{N_{S}} Q_{\phi, r i}^{S, n *} \boldsymbol{\Phi}_{\Gamma, r}^{S, 0 *}\right)\left(\sum_{t=1}^{N_{S}} Q_{\phi, t j}^{S, n} \boldsymbol{F}_{j, t}^{S, 0}\right) \\
=\sum_{r=1}^{N_{S}} \sum_{t=1}^{N_{S}} Q_{\phi, r i}^{S, n *} \boldsymbol{\Phi}_{\Gamma, r}^{S, 0 *} Q_{\phi, t j}^{S, n} \boldsymbol{F}_{j, t}^{S, 0}
\end{gathered}
$$

Because of the orthogonality of eigenvectors with different interblade phase angles, only terms in which $\boldsymbol{\Phi}_{\Gamma, r}^{S, 0}$ and $\boldsymbol{F}_{j, t}^{S, 0}$ have the same interblade phase angle will be nonzero in Eq. (18). The aerodynamic stiffness matrix in the tuned structure-only system modal coordinates can be derived in the same way as Eq. (16). One obtains

$$
\boldsymbol{K}^{a, n}=\boldsymbol{P}^{n *} \hat{\boldsymbol{A}}^{M, n} \boldsymbol{P}^{n}
$$

One may note that the tuned system can be calculated using the same method as for the mistuned system described earlier, by setting the mistuning to zero. However, each tuned mode shape $\boldsymbol{\Phi}_{\Gamma, r}^{S, 0}$ is related to $N_{S}$ different frequencies [in Eq. (18)], whereas every tuned mode shape $\boldsymbol{\Phi}_{\Gamma, i}^{S, n}$ is related to only one frequency in the method shown in Eqs. (15) and (16) for the tuned case. Thus, the method based on Eqs. (15) and (16) saves a large amount of computation time compared to the method based on Eqs. (17-19), but it is valid only for tuned cases.

\section{Case Study}

The structural ROM model has been validated by comparing the results with MSC-NASTRAN [8], and the aerodynamic code has been validated by comparing the results with several other unsteady CFD codes and experiment results $[16,31,32]$ for cascade flows. An industrial bladed disk with 26 sectors shown on the left in Fig. 1 was investigated. The complete FEM model has 1,306,500 DOF, and each blade has 21,582 DOF, with 990 DOF at the interface between the blade and the disk. The large number of interface DOF makes this case study challenging because the inclusion of the boundary constraint modes is practically impossible. Figure 2 shows the natural frequencies vs nodal diameter numbers for the tuned system. The frequency range from 1300 to $1700 \mathrm{~Hz}$ was studied. The frequency range investigated covers the second group (first torsion: 1T) of system modes. The rotation speed of interest is determined using the Campbell diagram shown in Fig. $\underline{3}$, and 15 layers are used for the aerodynamic calculations. The upstream far-field steady Mach number near the hub is 0.4 , and the reduced frequency (based on half chord and inlet velocity near the hub) is approximately 1.45 near the hub. For brevity, the method using the cantilever-blade normal modes is referred to as the blade mode method. The method using the system modes is referred to as the system mode method. Finally, the uncoupled and the coupled results using the system mode method are referred to as one-step results and converged results. Small mistuning of Young's modulus $E$ of every blade is considered. Table 1 shows the employed mistuning patterns $\left(\delta_{n}^{e}\right)$ and the corresponding blades. The standard deviation of $\delta_{n}^{e}$ is $2.7 \%$ and the mean value is close to zero. The structural damping $\gamma$ is 0.001 . The number of DOF of the CMM model is 28. Finally, five cantileverblade normal modes and 990 blade-disk boundary constraint modes are used for the mistuning projection, but only the corresponding cantilever-blade normal mode (1T) is used for the aeroelastic

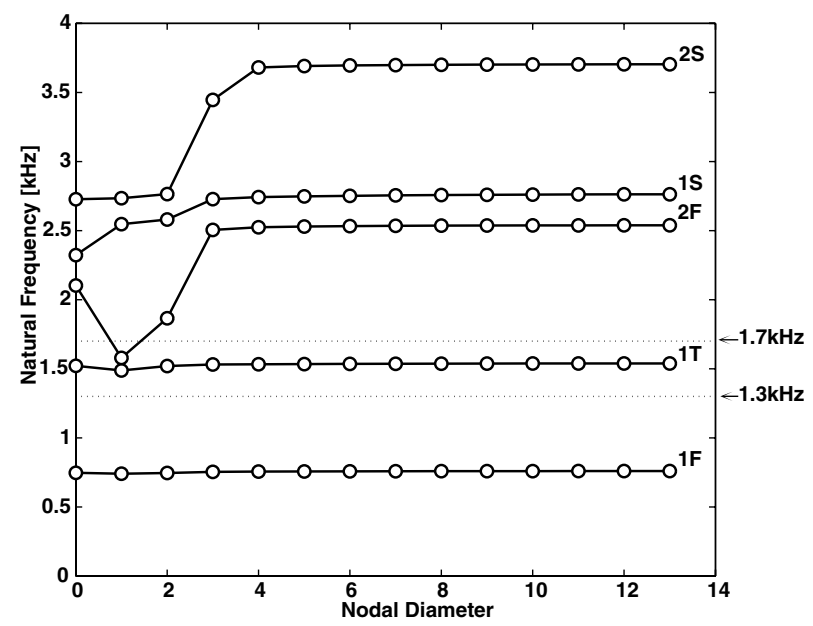

Fig. 2 Natural frequencies vs nodal diameter numbers for the tuned bladed disk. 


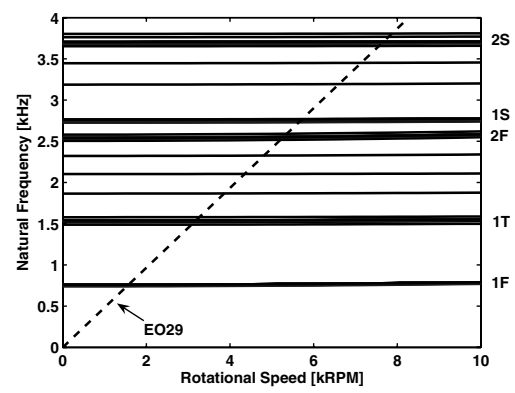

Fig. 3 Campbell diagram for the tuned bladed disk. EO stands for engine order.

calculation in the blade mode method (where not stated otherwise). As discussed in the following section, the bladed disk studied herein has relatively strong aerodynamic coupling, which may not be the case for a variety of other blisk operating conditions. Also, a strong sensitivity of aerodynamic forces to interblade phase angle is observed for the bladed disk studied.

\section{Free Vibration Problem}

Figure 4 shows the tuned and mistuned eigenvalue locus of the 1T system modes of the system in the complex plane $\left(\lambda=j \omega / \omega_{\text {ref }}\right.$, where $\omega_{\text {ref }}$ is a reference complex frequency). The imaginary part of an eigenvalue represents frequency, and the real part represents damping. A positive real part indicates that the damping is negative and that the system is unstable for the corresponding mode shape. The aeroelastic eigenvalues are distinct from the structure-only eigenvalues. The aerodynamic coupling is strong for this case study, as indicated by the fact that the largest aerodynamic damping value is close to 0.03 . Note that the regular pattern of the tuned aeroelastic eigenvalues is destroyed when mistuning is introduced. Compared to the tuned aeroelastic eigenvalues, the range of the real part of the mistuned aeroelastic eigenvalues narrows down, and the range of the imaginary part expands, which means that the mistuned frequencies spread out, and mistuning stabilizes the system. These results are in agreement with the well-known beneficial effect of mistuning on flutter (Pierre and Murthy [20], Pierre et al. [21]). For most of the tuned eigenvalues, the one-step results and converged results are almost identical for frequencies, but show notable differences in damping, which agrees with Gerolymos [23]. However, for the eigenvalue corresponding to the interblade phase angle index 3 , the converged result and the one-step result exhibit significant differences in both frequency and damping (as shown in Fig. 4). This indicates that for this traveling wave, the dependence of the unsteady aerodynamic force on the eigenvalue is complex. For the mistuned eigenvalues, the one-step and converged results show similar differences. The results using the blade mode method are close to the one-step results, whereas some of the tuned and mistuned eigenvalues have notable differences. In the following, a tuned system mode with a positive interblade phase angle corresponds to a traveling wave in the same direction of rotation.

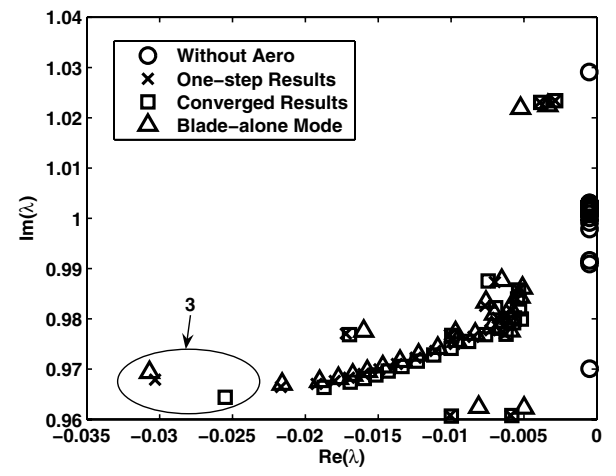

a) Tuned system
Table 1 Mistuning pattern in $E$ for the case study rotor

\begin{tabular}{lr}
\hline \hline Blade & $\delta_{n}^{e}, \%$ \\
\hline 1 & 5.06 \\
2 & 2.68 \\
3 & 2.30 \\
4 & -3.84 \\
5 & 4.27 \\
6 & -2.74 \\
7 & -4.29 \\
8 & -1.71 \\
9 & 0.81 \\
10 & 0.65 \\
11 & 1.20 \\
12 & -0.92 \\
13 & -3.17 \\
14 & 2.93 \\
15 & -1.67 \\
16 & -0.60 \\
17 & -3.65 \\
18 & -3.50 \\
19 & 0.03 \\
20 & 0.15 \\
21 & 3.27 \\
22 & 2.76 \\
23 & -0.62 \\
24 & -2.06 \\
25 & 0.91 \\
26 & -3.65 \\
\hline \hline
\end{tabular}

Pierre et al. [21] showed that the aeroelastic mode shapes transit from constant interblade phase angle (or extended) modes to localized modes when mistuning increases. Also, there are numerous mode crossings in the transition region, which are caused by the frequency switching for different mistuned modes. This is demonstrated in Fig. 5, which shows the fourth and fifth mistuned aeroelastic modes. The modes on the left column are the results using the blade mode method. The modes on the middle column are the one-step results using the system mode method. The modes on the right are the structure-only modes. Interestingly, the fourth mode on the left column is similar to the fifth mode on the middle column. Also, the fifth mode on the right column is similar to the fourth mode on the middle column. This indicates that the mistuned aeroelastic mode crossings are very complex in the transition region. For these two sets of results, although the fourth and fifth mistuned modes are similar, the mistuned mode crossing patterns are different because the frequency orders of these two modes are switched. However, the mistuned structure-only modes are totally different from the mistuned aeroelastic modes. In fact, for some structure-only modes, no similar modes can be found in the aeroelastic modes. For example, Fig. 6 shows the modal assurance criterion (MAC) numbers between the third structure-only mistuned mode and the aeroelastic mistuned modes using the blade mode method and the system mode method. The MAC number $\mathrm{MAC}_{c d}$ shows the linear dependence of two different modes $\boldsymbol{\Psi}_{c}$ and $\boldsymbol{\Psi}_{d}$, and is defined as [33]

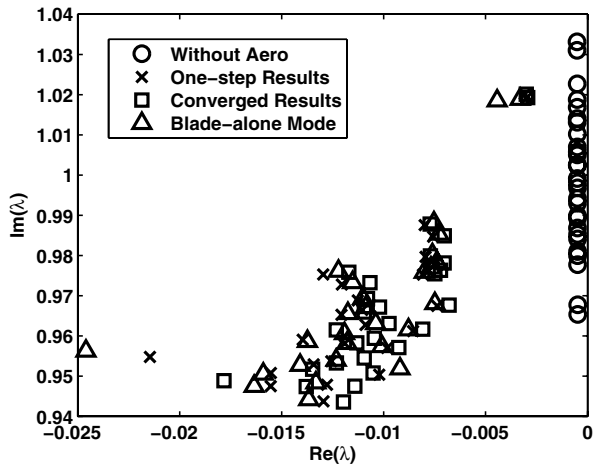

b) Mistuned system

Fig. 4 Eigenvalues of the 1T system modes with structural damping $\gamma$ of 0.001 . 
4-th mode

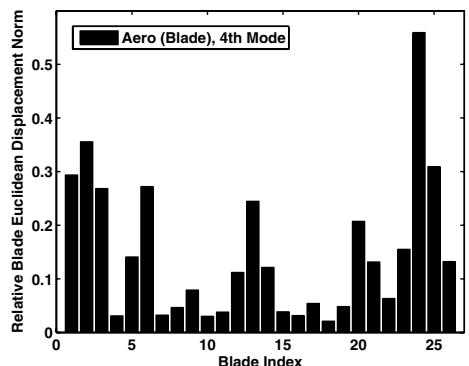

a) Blade mode results

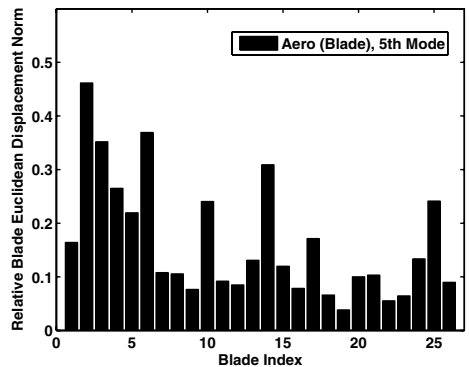

d) Blade mode results

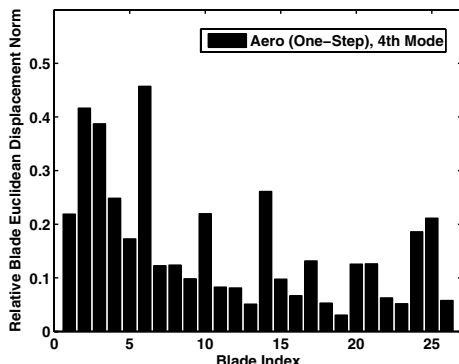

b) One-step results

5-th mode

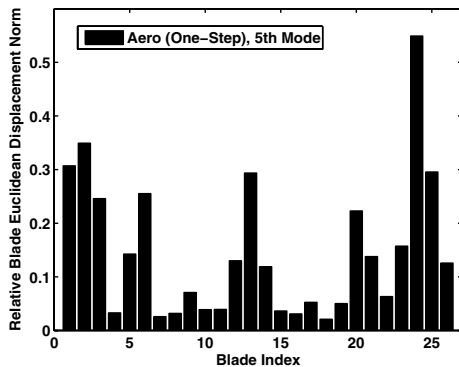

e) One-step results

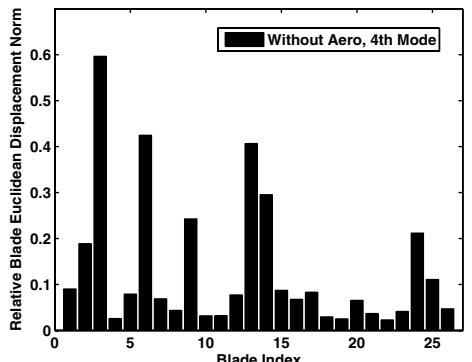

c) Structure-only mode

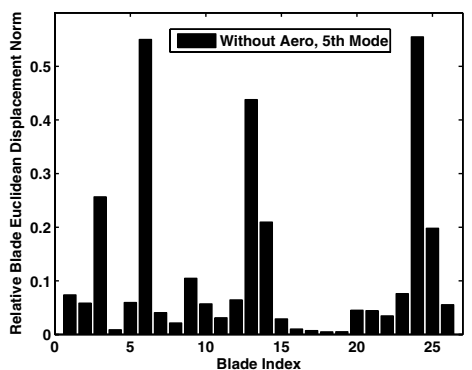

f) Structure-only mode

Fig. 5 Fourth and fifth mistuned mode shape amplitudes in the 1T frequency range.

$$
\operatorname{MAC}_{c d}=\frac{\left\|\left(\boldsymbol{\Psi}_{c}\right)^{*} \cdot\left(\boldsymbol{\Psi}_{d}\right)\right\|_{2}^{2}}{\left\|\boldsymbol{\Psi}_{c}\right\|_{2}^{2} \cdot\left\|\boldsymbol{\Psi}_{d}\right\|_{2}^{2}}
$$

The range of the MAC number is from 0 to 1 , and a MAC number of 1 corresponds to two modes that are linearly dependent. As shown in Fig. 6 , the highest MAC number is only about 0.3 , which confirms the clear difference between structure-only and aeroelastic modes.

The aeroelastic mistuned modes are less localized compared to the structural mistuned modes. Wei and Pierre [2] showed that not only the mistuning level, but also the interblade coupling is important for a mistuned mode to be localized. In this case, the additional aerodynamic interblade coupling affects the severity of mode localization. For example, Fig. 7 shows the MAC numbers between the 10th converged aeroelastic mistuned mode and the mistuned modes using other methods. The 10th and 11th one-step aeroelastic modes, as well as the 11th mode using the blade mode method, have the largest MAC numbers close to 0.6 with this mode. Also, there is no similar structural mode for this aeroelastic mode. Figure 8 shows the magnitude of this mode (Fig. 8a) and the 10th (Fig. 8b) and 11th (Fig. 8c) one-step aeroelastic modes. Although the M $\overline{A C}$ numbers between the 10th mistuned converged mode and these two one-step

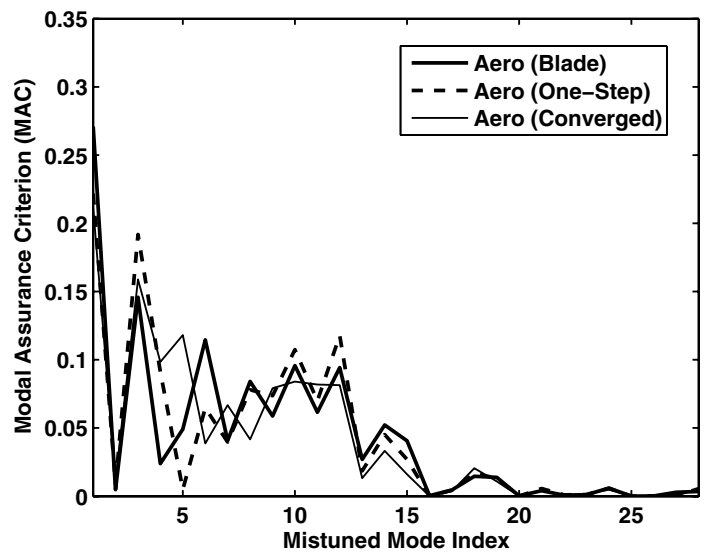

Fig. 6 MAC numbers between the third structure-only mistuned mode and aeroelastic mistuned modes. modes are the largest ones, there are significant differences between the blade amplitude patterns of these three modes.

\section{Forced Response}

The tuned and mistuned forced responses of the system in the 1T system mode frequency range, subjected to an engine order 29 excitation, are shown in Fig. 9. The applied forces are unit loads on one of the nodes at the tip of each blade. The aeroelastic peak frequencies are smaller than the structure-only peak frequencies, which can be predicted from Fig. 4 a because an engine order 29 excitation corresponds to the same interblade phase angle as interblade phase angle index 3 . In general, the aerodynamic stiffness matrix should be recalculated for every excitation frequency; however, only one aerodynamic stiffness matrix, calculated for the eigenvalue problem, was used herein. The fluid-structure coupling introduces additional damping into the system, so that the tuned and mistuned aeroelastic peak forced response amplitudes are also smaller than those observed for the structure-only system. Also, the additional aerodynamic coupling leads to the disappearance of several mistuned forced response peaks. The results using the blade mode method are similar to the results using the system mode

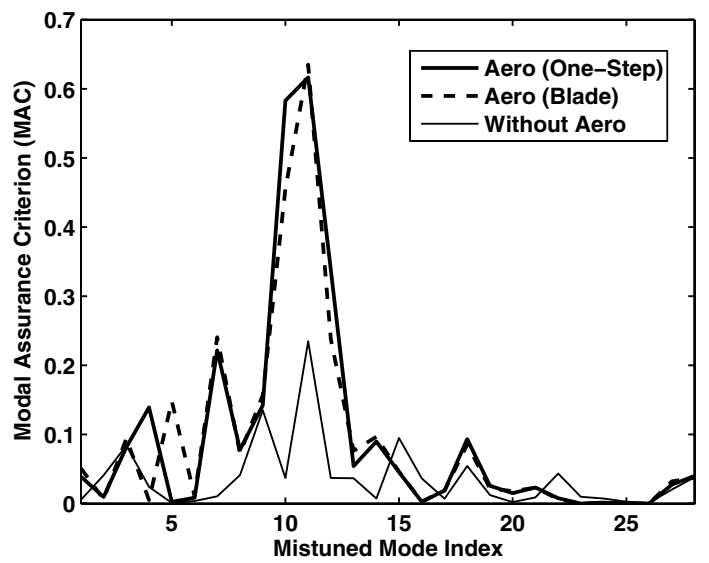

Fig. 7 MAC numbers between the 10th converged aeroelastic mistuned mode and other modes. 


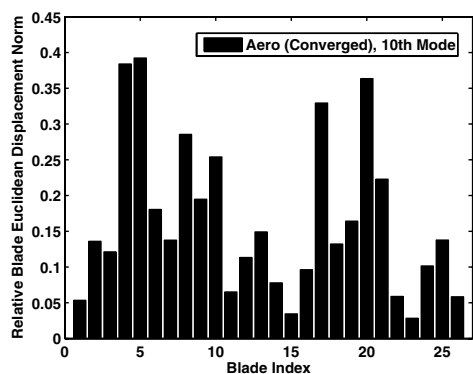

a) 10th Converged aeroelastic mode

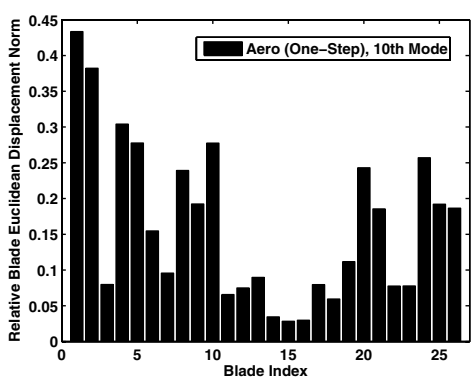

b) 10th One-step aeroelastic mode

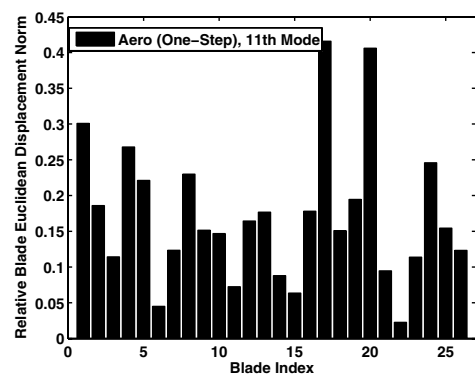

c) 11th One-step aeroelastic mode

Fig. 8 Mistuned aeroelastic mode shape amplitudes.

method. Nonetheless, it is interesting to note that the resonance frequency where the maximum mistuned aeroelastic forced response occurs is different for the results using the blade mode method and the system mode method.

The amplification factor, defined as the ratio between the mistuned and tuned peak values, is 1.12 for the structure-only case, and it is $1.87,1.93$, and 1.94 for the one-step result, converged result, and the result using the blade mode method. The large increase of the amplification factor due to the inclusion of aerodynamic coupling can be predicted from Fig. 4. As shown in Fig. 4 a, the tune mode corresponding to interblade phase angle index $\overline{3}$ has the largest aerodynamic damping. A mistuned aeroelastic mode can be viewed as a linear combination of tuned aeroelastic modes. Therefore, the damping of a mistuned aeroelastic mode can also be viewed as a linear combination of the damping of tuned aeroelastic modes. Hence, the beneficial effect of mistuning on flutter (as shown in Fig. $4 \mathrm{~b}$ ) is observed because the range of the mistuned aerodynamic damping is smaller than the range of the tuned aerodynamic damping. Thus, all the values of the mistuned aerodynamic damping are smaller than the tuned aerodynamic damping corresponding to the interblade phase angle index 3 . Hence, the amplification factor is larger because the mistuned aerodynamic damping is smaller.
The 95 percentile response levels shown in Fig. 10 are calculated using Monte Carlo simulations to determine statistically the likely maximum forced response amplification factor due to mistuning. A number of 1000 mistuning patterns are used for each mistuning level. Note that the Monte Carlo simulations here assume that the aerodynamic stiffness matrix is the same as the one obtained using the mistuning pattern shown in Table $\underline{1}\left(\delta_{n}^{e}\right)$. In general, the aerodynamic stiffness matrix may be different for distinct mistuning patterns if the system mode method is used. As shown in Fig. 10, the aeroelastic 95 percentile response levels are smaller than the structure-only response when the mistuning level is small. This indicates that the sensitivity of the amplification factor to mistuning is decreased by the aerodynamic coupling. When the mistuning level becomes larger, the aeroelastic 95 percentile response levels overpass the structure-only response and increase to a larger value, around 3. This is consistent with Fig. 9. To demonstrate the effect of different damping values for different tuned system modes (as in the case of aerodynamic damping), two 95 percentile response levels are also plotted in Fig. 10. The first one is referred to as the varied structural damping case and the second one is referred to as the very large structural damping case. In the varied structural damping case, no aeroelastic coupling is considered and the structural damping $\gamma$

Tuned System
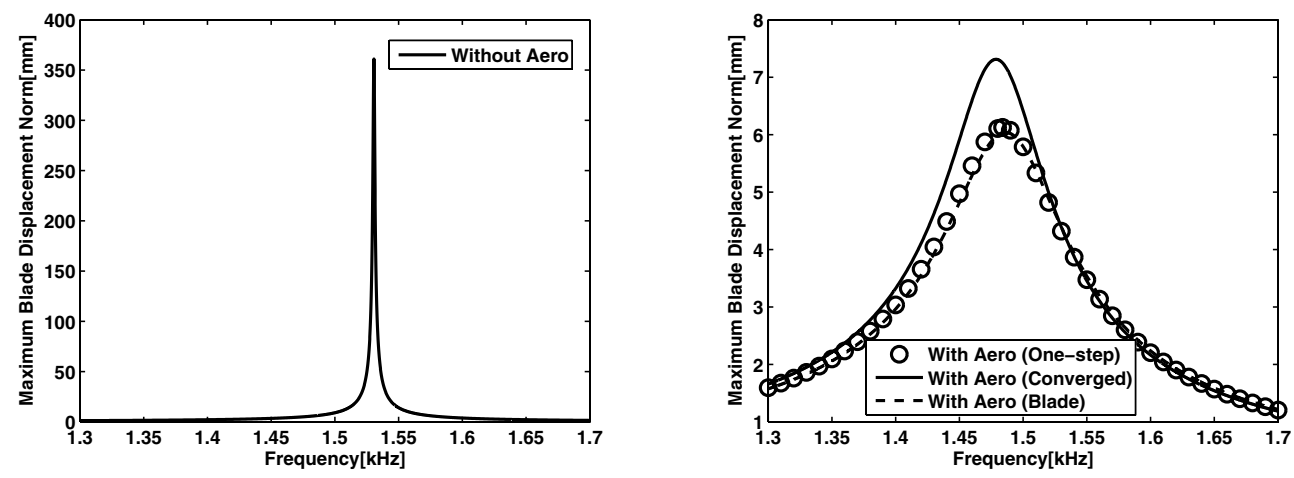

a) Structure-only results

b) Aeroelastic results

Mistuned System

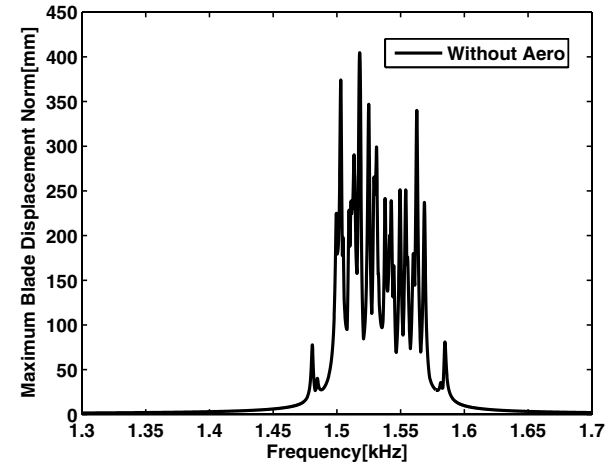

c) Structure-only results

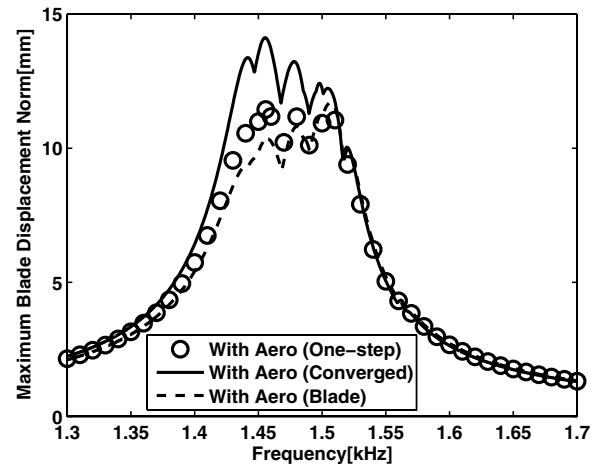

d) Aeroelastic results

Fig. 9 Forced response for engine order 29 excitation. 


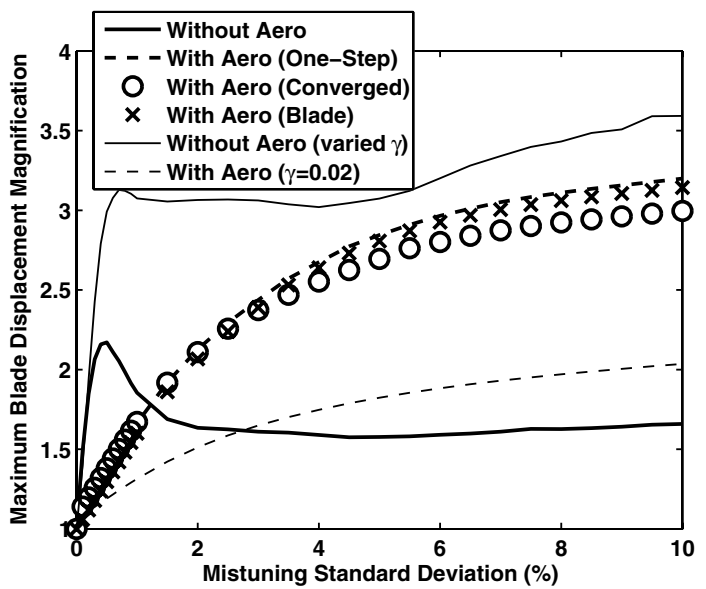

Fig. 10 Ninety-five percentile response levels for the engine order 29 excitation.

related to nodal diameter 3 modes is 0.003 , whereas the structural damping $\gamma$ is 0.001 for the other modes. In the very large structural damping case, the aeroelastic coupling using the system mode method with converged results is included, and the structural damping $\gamma$ is set to 0.02 . Compared to the structure-only result with fixed $\gamma=0.001$, the sensitivity of the amplification factor to mistuning does not change significantly in the varied structural damping case. However, for this case, when the mistuning standard deviation becomes larger than $0.5 \%$, the amplification factor becomes larger than 3 . Compared to the aeroelastic results with $\gamma=0.001$, the amplification factor is always smaller than 2 in the very large structural damping case because the very large structural damping decreases the relative difference between the mistuned aeroelastic damping and the tuned aeroelastic damping corresponding to the interblade phase angle index 3 . Note that in the very large structural damping case, the sensitivity of the amplification factor to mistuning from the aeroelastic calculation is still smaller than the structure-only results.

\section{Accuracy of the Blade Mode Method}

For brevity, the cantilever-blade normal modes are referred to as the blade modes in this section. As shown in the preceding subsection, the results using the blade mode method has notable differences compared to the results using the system mode method. There are at least two possible reasons for these differences: one is the difference in the vibration frequencies used in the aerodynamic calculation (system natural frequencies are used in the system mode method, and natural frequencies of cantilever-blade normal modes are used in the blade mode method), the other is the difference in the mode shapes. To account for the difference in the vibration frequencies, a similar iterative process to the system mode method can be developed. To account for the difference in the mode shapes, the neglected constraint modes can be included. However, the inclusion of the constraint modes using the modal projection similar to Eq. (11) is practically formidable for the aerodynamic calculations because of the large number of constraint modes. For example, for this case study, the required computation time is about $4.7 \mathrm{~s}$ on a SunBlade-1000 machine for a single passage of a layer with respect to a specific mode shape and eigenvalue. Then, using the blade mode method with just one blade mode requires about $0.5 \mathrm{~h}$ of computation time because there are 15 layers and 26 different interblade phase angle for every blade mode. Usually, the computational time for numerical simulations cannot be estimated using a simple multiplicative relation. However, here that is possible because the unsteady code used in this paper is a frequency domain code, and the computation time of the structural ROM code as well as the computation time of the steady aerodynamic calculation are negligible compared to the computation time of the unsteady aerodynamic calculation. Hence, using the blade mode method with one blade mode and 990 constraint modes would cost approximately
$500 \mathrm{~h}$. Using the system mode method with 26 tuned system modes consumes about $0.5 \mathrm{~h}$ per iteration step for the tuned system, whereas the required computation time is about $13 \mathrm{~h}$ per iteration step for the mistuned system (because the unsteady calculation is needed for 26 different eigenvalues for every tuned system mode).

To investigate the effect of neglecting the constraint modes, some artificial blade modes are generated to calculate the aerodynamic stiffness shown in Eq. (7). These artificial blade modes are either the system mode related to the nodal diameter zero, or the corresponding combined blade modes with or without the constraint modes [shown in Eqs. (4) and (9). For example, for the $1 \mathrm{~T}$ system mode frequency range, these generated artificial blade modes replace the original 1T blade mode to conduct the aerodynamic calculation, and then use the modal projection shown in Eq. (11) to calculate the aerodynamic stiffness matrix. Figure 11 shows the resulting aerodynamic stiffness coefficients [the diagonal elements of $\tilde{\mathbf{A}}$ in Eq. (7)] using these artificial modes generated from the system mode and using the second, the first two, the first four, and the first ten blade modes. The MAC number between the system mode and the corresponding 1T blade mode is 0.992 , which means that they are very close to linearly dependent. However, the results using the second blade mode without the constraint modes are different from the results using the system mode. The inclusion of other blade modes changes the real part of the aerodynamic stiffness matrix, but does not change the imaginary part. Nonetheless, the results with the constraint modes are in good agreement with the results using the system mode, especially when other blade modes are also included. Note that in Fig. 11, a strong sensitivity of aerodynamic stiffness coefficients on the interblade phase angle is observed, particularly for interblade phase angle index 3. It is believed that a fluid instability exists in this region for the case studied. The difference between the one-step and converged results shown in Fig. $\underline{4}$ can be caused by such a fluid instability, which is sensitive to frequency. Figure 12 shows the tuned forced responses to the engine order 5 excitation. The aerodynamic stiffness matrices are obtained using the artificial modes generated from the system mode and the first two blade modes (with and without the constraint modes). Significant differences in the peak forced response values and the resonance frequencies are observed between the results using the system mode and the first blade mode without the constraint modes. The inclusion of the constraint modes yields almost identical results with the results obtained using the system modes.

In principle, the blade mode method with all the constraint modes would be more accurate than the system mode method because the constraint modes represent all the physical coupling at the interface, whereas only the modal coupling between the select tuned system modes is considered in the system mode method. That would be the case only if all the constraint modal coordinates are kept in the final ROM modal space. However, the size of the final ROM modal space and the required time for the aerodynamic calculation would become extremely large. Hence, the system mode method combined with the CMM model is a very good compromise between accuracy and computation time.

\section{Conclusions}

In this study, the effects of aerodynamic coupling on the vibration response of bladed disks were investigated. An iterative method using the tuned system modes to calculate the aerodynamic coupling was developed. The results were compared with structure-only results and the results using the blade-alone normal modes. A bladed disk and aeroelastic configuration that exhibits relatively strong aerodynamic coupling was found in the case study herein. Also, the aerodynamic stiffness forces are strongly sensitive to interblade phase angle for the bladed disk studied.

For the case studied, it was shown that aeroelastic coupling may change the tuned and mistuned forced responses. Also, the statistical 95 percentile response levels are affected significantly by aerodynamic forces. Furthermore, the mistuned mode shapes change when aeroelastic coupling is introduced. 


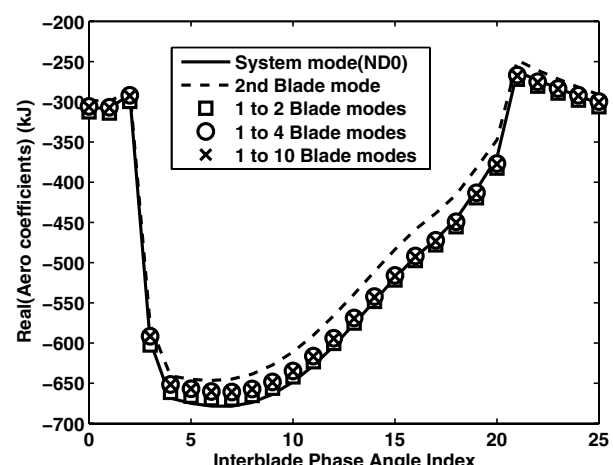

a) Real part (without constraint modes)

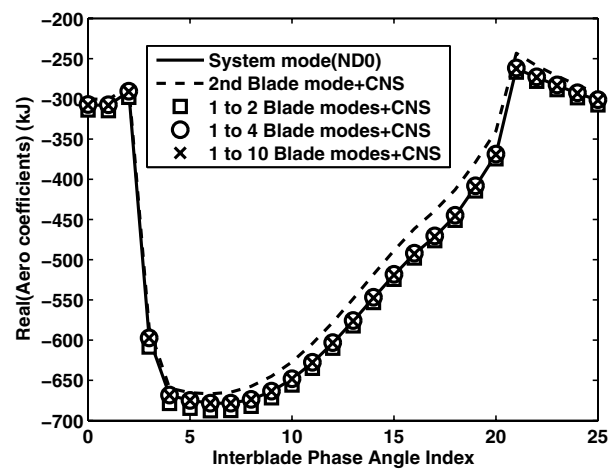

c) Real part (with constraint modes)

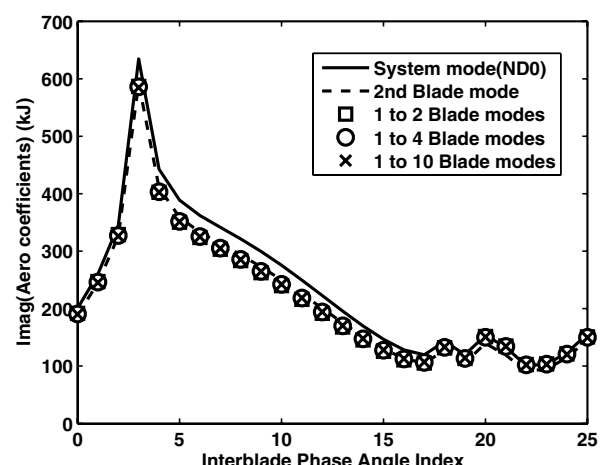

b) Imaginary part (without constraint modes)

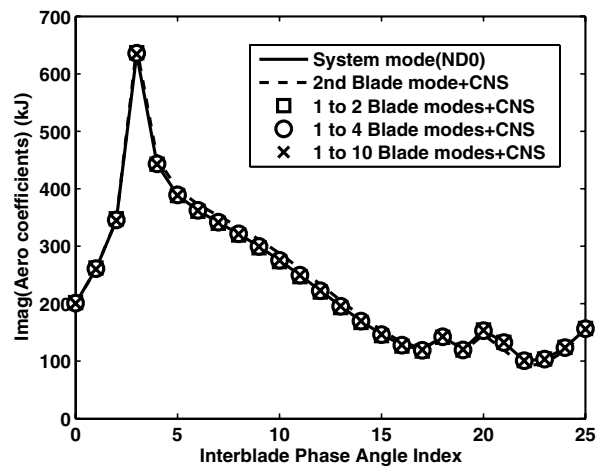

d) Imaginary part (with constraint modes)

Fig. 11 Aerodynamic stiffness coefficients; CNS stands for constraint.

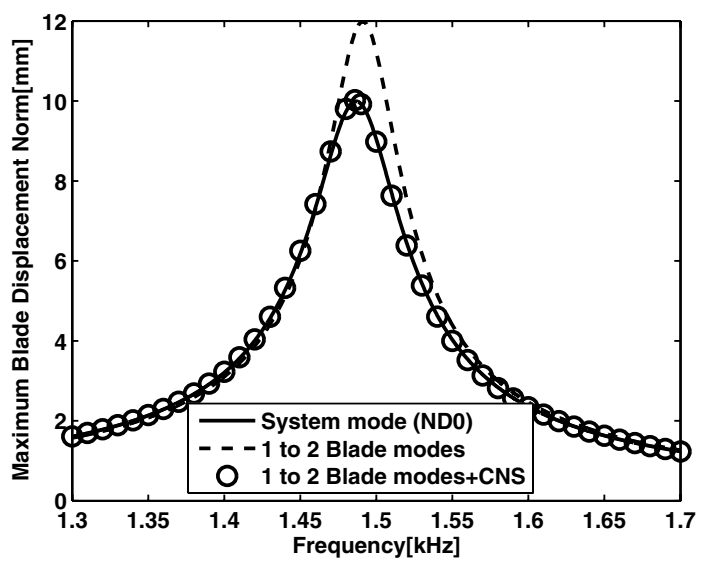

Fig. 12 Tuned forced response to engine order 5 excitation; CNS stands for constraint.

Mistuned mode crossing patterns are different for the results using the blade and system mode methods for the cases studied. Also, the one-step and converged aeroelastic coupling using the system mode methods yield similar results for the cases studied. However, when aeroelastic eigenvalues and mode shapes are of critical interest, multiple iterations and converged results should be used.

The constraint modes are necessary for obtaining accurate results when using the blade mode method for the case studied. However, the large number of constraint modes makes the computation time much larger than that of using the system mode method.

\section{Acknowledgments}

The authors gratefully acknowledge the support of the GUIde Consortium on Blade Durability at Carnegie Mellon University and the Air Force Office of Scientific Research.

\section{References}

[1] Tobias, S. A., and Arnold, R. N., "The Influence of Dynamical Imperfection on the Vibration of Rotating Disks," Proceedings of the Institution of Mechanical Engineers, Vol. 171, 1957, pp. 669-690.

[2] Wei, S. T., and Pierre, C., "Localization Phenomena in Mistuned Assemblies with Cyclic Symmetry, Part 1: Free Vibration," Journal of Vibration, Acoustics, Stress, and Reliability in Design, Vol. 110, No. 4, 1988, pp. 429-438.

[3] Wei, S. T., and Pierre, C., "Localization Phenomena in Mistuned Assemblies with Cyclic Symmetry, Part 2: Forced Vibrations," Journal of Vibration, Acoustics, Stress, and Reliability in Design, Vol. 110, No. 4, 1988, pp. 439-449.

[4] Griffin, J. H., and Hoosac, T. M., "Model Development and Statistical Investigation of Turbine Blade Mistuning," Journal of Vibration, Acoustics, Stress, and Reliability in Design, Vol. 106, No. 2, 1984, pp. 204-210.

[5] Castanier, M. P., Òttarsson, G., and Pierre, C., "A Reduced Order Modeling Technique for Mistuned Bladed Disks," Journal of Vibration and Acoustics, Vol. 119, No. 3, 1997, pp. 439-447.

[6] Bladh, R., Castanier, M. P., and Pierre, C., "Component-Mode-Based Reduced Order Modeling Techniques for Mistuned Bladed Disks, Part 1: Theoretical Models," Journal of Engineering for Gas Turbines and Power, Vol. 123, No. 1, 2001, pp. 89-99.

[7] Bladh, R., Castanier, M. P., and Pierre, C., "Component-Mode-Based Reduced Order Modeling Techniques for Mistuned Bladed Disks, Part 2: Applications," Journal of Engineering for Gas Turbines and Power, Vol. 123, No. 1, 2001, pp. 100-108.

[8] Lim, S., Bladh, R., Castanier, M. P., and Pierre, C., "A Compact, Generalized Component Mode Mistuning Representation for Modeling Bladed Disk Vibrations," Proceedings of the 44th AIAA/ASME/ASCE/ AMA Structures, Structural Dynamics and Material Conference, Vol. 2, AIAA, Reston, VA, 2003, pp. 1359-1380.

[9] Yang, M. T., and Griffin, J. H., "A Normalized Model Eigenvalue Approach for Resolving Model Interaction," Journal of Engineering for Gas Turbines and Power, Vol. 119, No. 3, 2001, pp. 647-650.

[10] Yang, M. T., and Griffin, J. H., "A Reduced-Order Model of Mistuning Using a Subset of Nominal System Modes," Journal of Engineering for Gas Turbines and Power, Vol. 123, No. 4, 2001, pp. 893-900.

[11] Feiner, D. M., and Griffin, G. H., "A Fundamental Model of Mistuning for a Single Family of Modes," Journal of Turbomachinery, Vol. 124, No. 4, 2002, pp. 597-605. 
[12] Petrov, E. P., Sanliturk, K. Y., and Ewins, D. J., "A New Method for Dynamic Analysis of Mistuned Bladed Disks Based on the Exact Relationship Between Tuned and Mistuned Systems," Journal of Engineering for Gas Turbines and Power, Vol. 124, No. 3, 2002, pp. 586-597.

[13] Hall, K. C., "Deforming Grid Variational Principle for Unsteady Small Disturbance Flows in Cascades," AIAA Journal, Vol. 31, No. 5, 1993, pp. 891-900.

[14] Hall, K. C., and Lorence, C. B., "Calculation of Three-Dimensional Unsteady Flows in Turbomachinery Using the Linearized Harmonic Euler Equations," Journal of Turbomachinery, Vol. 115, No. 4, 1993, pp. 800-809.

[15] Chaviaropoulos, P. K., and Hansen, M. O. L., "Investigating ThreeDimensional and Rotational Effects on Wind Turbine Blades by Means of a Quasi-3D Navier-Stokes Solver," Journal of Fluids Engineering, Vol. 22, No. 2, 2000, pp. 330-336.

[16] Fransson, T. H., Jöcker, M., Bölcs, A., and Ott, P., "Viscous and Inviscid Linear/Nonlinear Calculations Versus Quasi-Three-dimensional Experimental Cascade Data for a New Aeroelastic Turbine Standard Configuration," Journal of Turbomachinery, Vol. 121, No. 4, 1999, pp. 717-725.

[17] Bell, D. L., and He, L., "Three-Dimensional Unsteady Flow for an Oscillating Turbine Blade and the Influence of Tip Leakage," Journal of Turbomachinery, Vol. 122, No. 1, 2000, pp. 93-101.

[18] Kaza, K. R. V., and Kielb, R. E., "Flutter of Turbofan Rotors with Mistuned Blades," AIAA Journal, Vol. 22, No. 11, 1984, pp. 16181625.

[19] Kaza, K. R. V., and Kielb, R. E., "Vibration and Flutter of Mistuned Bladed-Disk Assemblies," Journal of Propulsion and Power, Vol. 1, No. 5,1985 , pp. 336-344.

[20] Pierre, C., and Murthy, D., "Aeroelastic Model Characteristics of Mistuned Blade Assemblies: Mode Localization and Loss of Eigenstructure," AIAA Journal, Vol. 30, No. 10, 1992, pp. 2483-2496.

[21] Pierre, C., Smith, T. E., and Murthy, D., "Localization of Aeroelastic Modes in Mistuned High-Energy Turbines," Journal of Propulsion and Power, Vol. 10, No. 3, 1994, pp. 318-328.

[22] Sadeghi, M., and Liu, F., "Computation of Mistuning Effects on Cascade Flutter," AIAA Journal, Vol. 39, No. 1, 2001, pp. 22-28.

[23] Gerolymos, G. A., "Coupled Three-Dimensional Aeroelastic Stability Analysis of Bladed Disks," Journal of Turbomachinery, Vol. 115, No. 4, 1993, pp. 791-799.

[24] Moyroud, F. G., Jacquet-Richardet, G.., and Fransson, T., "A Model Coupling for Fluid and Structure Analyses of Turbomachine Flutter:
Application to a Fan Stage,” ASME Paper 96-GT-335, 1996, pp. 110 .

[25] Seinturier, E., Dupont, C., Berthillier, M., and Dumas, M., "A New Aeroelastic Model for Mistuned Bladed Disks," Proceedings of the 43rd AIAA/ASME/ASCE/AHS/ASC Structures, Structural Dynamics, and Materials Conference, Vol. 4, AIAA, Reston, VA, 2002, pp. 26282638.

[26] Kielb, R. E., Feiner, D. M., Griffin, J. H., and Miyakozawa, T., "Probabilistic Analysis of Mistuned Bladed Disks and Blisks with Aerodynamic and FMM Structural Coupling," Proceedings of the 9th National Turbine Engine HCF Conference, Universal Technology Corporation, Dayton, OH, 2004, pp. 1-10.

[27] Kielb, R. E., Feiner, D. M., Griffin, J. H., and Miyakozawa, T., "Flutter of Mistuned Bladed Disks and Blisks with Aerodynamic and FMM Structural Coupling," Proceedings of ASME Turbo Expo 2004, Vol. 6, ASME, New York, 2004, pp. 573-579.

[28] Cedar, R. D., and Stow, P., "The Addition of Quasi-Three-Dimensional Terms into a Finite Element Method for Transonic Turbomachinery Blade-to-Blade Flows," International Journal for Numerical Methods in Fluids, Vol. 5, No. 2, 1985, pp. 101-114.

[29] Bateman, H., "Irrotational Motion of a Compressible Fluid," Proceedings of the National Academy of Sciences of the United States of America, Vol. 16, No. 12, 1930, pp. 816-825.

[30] Horn, R., and Johnson, C., Topics in Matrix Analysis, Cambridge Univ. Press, Cambridge, England, 1991.

[31] Fransson, T. H., and Verdon, J. M., "Updated Report on Standard Configurations for Unsteady Flow Through Vibrating Axial-Flow Turbomachine Cascades: Status as of July 1991," Tech. rept., Royal Institute of Technology, Stockholm, Sweden and United Technologies Research Center, East Hartford, CT, 1992, http://www.energy.kth.se/ proj/projects/Markus\%20Joecker/STCF/STCF1to10/Documents/ SC2110.92update.pdf [cited 19 Dec. 2006].

[32] Epureanu, B. I., Dowell, E. H., and Hall, K. C., "Mach Number Influence on Reduced-Order Models of Inviscid Potential Flows in Turbomachinery," Journal of Fluids Engineering, Vol. 124, No. 4, 2002, pp. 977-987.

[33] Allemang, R. J., "The Modal Assurance Criterion-Twenty Years of Use and Abuse," Journal of Sound and Vibration, Vol. 37, No. 8, 2003, pp. 14-23. 